\title{
Do Toll-like Receptors Play a New Role as a Biomarker of Irritable Bowel Syndrome?
}

\author{
Hyun Jin Kim \\ Department of Internal Medicine, Gyeongsang National University Changwon Hospital, Changwon, Gyeongsangnam-do, Korea
}

\author{
Article: Expression of toll-like receptors, pro-, and anti-inflammatory cytokines in relation to gut microbiota in irritable bowel \\ syndrome: the evidence for its micro-organic basis \\ Shukla R, Ghosahl U, Ranjan P, Ghoshal UC \\ (J Neurogastroenterol Motil 2018;24:628-642)
}

Traditionally, irritable bowel syndrome (IBS) is defined that is a functional bowel disorder characterized by abdominal pain or discomfort and altered bowel habits in the absence of detectable structural abnormalities. ${ }^{1}$ However, now the definition of IBS is that it is a common chronic functional gastrointestinal (GI) disorder characterized by recurring abdominal pain, bloating, loose or frequent stools and/or constipation in the absence of structural, major inflammatory, or biochemical abnormalities. ${ }^{2}$ The new definition of IBS is a concept that also involves low grade mucosal inflammation, altered mucosal permeability, and microbiota-host interactions that modulate low grade immune activation. ${ }^{3}$

Based on varied recent studies, ${ }^{4-8}$ low-grade mucosal inflammation, altered bacterial microbiota and/or altered intestinal permeability play a major role in the pathophysiology of IBS. Even in the normal mucosa, the epithelial mucosa between the gut microbiota and the human intestine play the important barrier role for mucosal immune protection through the innate immune response. ${ }^{6-8}$ Innate immunity in the GI tract include the anatomical barriers (peristalsis, gastric acid, bile acids, digestive enzyme, thiocyanate, gut flora, etc), complement system, inflammatory mediated white blood cells (neutrophils, basophils, and eosinophils), and other inflammatory mediated cells (natural killer cells, macrophages, mast cells, dendritic cells, etc). Pattern recognition receptors, a part of the innate immunity response, such as toll-like receptors (TLRs) and nucleotide-binding oligomerization domain-like receptors, mediate this interaction between pathogen-associated molecular patterns (PAMPs) and the human body. ${ }^{9}$

The TLRs has a family of type I transmembrane receptors (extracellular leucine-rich repeat domain and intracellular toll/IL-1 receptor domain). There are at least 10 TLRs and each TLR seems to have a distinct function in recognition of innate immunity. ${ }^{9}$ The most TLR ligands are conserved microbial products (eg, PAMPs), each individual TLR can recognize unrelated ligands. Some TLRs require accessory proteins to recognize their ligands. The TLR and IL-1 receptor (IL-1R)-family members share several signaling components, including the adaptor (myeloid differentiation primary response 88), toll-interacting protein, the protein kinase (IL-1Rassociated kinase), and TNF receptor-associated factor 6. TLRs sense the presence of infection through recognition of PAMPs. ${ }^{9}$

In this issue of Journal of Neurogastroenterology and Motility,

Received: September 11, 2018 Revised: None Accepted: September 13, 2018

↔ This is an Open Access article distributed under the terms of the Creative Commons Attribution Non-Commercial License (http://creativecommons. org/licenses/by-nc/4.0) which permits unrestricted non-commercial use, distribution, and reproduction in any medium, provided the original work is properly cited.

${ }^{*}$ Correspondence: Hyun Jin Kim, MD Department of Internal Medicine, Gyeongsang National University Changwon Hospital, 11 Samjeongja-ro, Seongsan-gu, Changwon, Gyeongsangnam-do 51472, Korea Tel: +82-55-214-3710, Fax: +82-55-214-3250, E-mail: imdrkim@naver.com 
Ghoshal et $\mathrm{al}^{10}$ reported that TLRs (2, 4, and 5), pro-inflammatory (IL-6, C-X-C motif chemokine ligand 11 [CXCL-11], C-X-C motif chemokine receptor 3 [CXCR-3]), and anti-inflammatory cytokine (IL-10) play a important relation to the gut microbiota in IBS. They evaluated TLRs expression in mRNA level in 47 patients with IBS (20 diarrhea-predominant IBS [IBS-D], 20 constipation-predominant IBS, and 7 unclassified IBS using the Rome III criteria) and 25 controls, and also investigated the expressions of TLR-4 and TLR-5, the quantitative real-time polymerase chain reaction of cytokines (IL-6 and IL-10), chemokine (CXCL-11), and its receptor (CXCR-3) in mRNA levels. This study was designed with the following objectives: (1) expression of TLR-2, 4, and 5 in colonic biopsies among patients with IBS and controls; (2) expression of pro-inflammatory (IL-6, CXCL-11, and CXCR-3), and anti-inflammatory cytokines (IL-10) in colonic biopsies among patients with IBS and controls; and (3) relationship between mRNA level of TLRs, cytokines and chemokines with the altered fecal microbiota published previously at these centers in Northern India. They found the mRNA levels of TLR-4 and TLR-5 were up-regulated in IBS, the expression of TLR-4 and TLR-5 at the protein level was higher in IBS-D. The mRNA level of IL-6, CXCL-11, and CXCR-3 was higher among IBS patients. Expression of IL-6, CXCL-11, and CXCR-3 was up-regulated and IL-10 was downregulated in IBS-D. Positive correlation was seen between TLR-4 and IL-6, CXCR-3 and CXCL-11, and IL-6 and CXCR-3. Stool frequency per week showed positive correlation with mRNA level of TLR-4 and CXCR-3, but inversely correlated with IL-10. Copy number of Lactobacillus and Bifidobacterium showed correlation with IL-10 in constipation-predominant IBS, while Gram-positive and Gram-negative bacteria showed correlation with CXCL-11 in IBS-D patients.

The results from Ghoshal et $\mathrm{al}^{10}$ serve as an important evidence for low-grade mucosal inflammation of GI tract mucosa. TLRs, also, are obviously related with activated immune activation and proinflammatory cytokines secretion. The altered gut microbiota has a sufficient role in altering the degree of mucosal inflammation and varied depending on the IBS subtypes. So, the active innate immune system caused by dysbiosis of gut microbiota may play an important role in initiation and exaggeration of visceral hyperalgesia of IBS. Little evidence is at present available regarding how PAMPs concentrations in the gut contents may be altered during the course of the IBS altering the expression of TLRs. It would like to be mentioned that possibility of various TLRs may potential as a biomakers of IBS.

\section{Financial support: None.}

\section{Conflicts of interest: None.}

\section{References}

1. Longstreth GF, Thompson WG, Chey WD, Houghton LA, Mearin F, Spiller RC. Functional bowel disorders. Gastroenterology 2006;130:1480-1491.

2. Chey WD, Kurlander J, Eswaran S. Irritable bowel syndrome: a clinical review. JAMA 2015;313:949-958.

3. Rodríguez-Fandiño O, Hernández-Ruiz J, Schmulson M. From cytokines to toll-like receptors and beyond - current knowledge and future research needs in irritable bowel syndrome. J Neurogastroenterol Motil 2010;16:363-373.

4. Ohman L, Isaksson S, Lindmark AC, et al. T-cell activation in patients with irritable bowel syndrome. Am J Gastroenterol 2009;104:1205-1212.

5. Cremon C, Gargano L, Morselli-Labate AM, et al. Mucosal immune activation in irritable bowel syndrome: gender dependence and association with digestive symptoms. Am J Gastroenterol 2009;104:392-400.

6. Barbara G, De Giorgio R, Stanghellini V, Cremon C, Corinaldesi R. A role for inflammation in irritable bowel syndrome? Gut 2002;51(supp 1):i41-i44

7. Kassinen A, Krogius-Kurikka L, Mäkivuokko H, et al. The fecal microbiota of irritable bowel syndrome patients differs significantly from that of healthy subjects. Gastroenterology 2007;133:24-33

8. Camilleri M, Gorman H. Intestinal permeability and irritable bowel syndrome. Neurogastroenterol Motil 2007;19:545-552.

9. Medzhitov R. Toll-like receptors and innate immunity. Nat Rev Immunol 2001;1:135-145.

10. Shukla R, Ghosahl U, Ranjan P, Ghoshal UC. Expression of toll-like receptors, pro-, and anti-inflammatory cytokines in relation to gut microbiota in irritable bowel syndrome: the evidence for its micro-organic basis. J Neurogastroenterol Motil 2018;24:628-642. 